\title{
Predicting Metal-binding Site Residues in Low-resolution Structural Models
}

\author{
Jaspreet Singh Sodhi', Kevin Bryson ${ }^{1}$, Liam J. McGuffin ${ }^{1}$ \\ Jonathan J. Ward ${ }^{1}$, Lorenz Wernisch ${ }^{2}$ and David T. Jones ${ }^{1 *}$
}

${ }^{1}$ Bioinformatics Unit

Department of Computer

Science, University College

London, Gower Street, London

WC1E 6BT, UK

${ }^{2}$ School of Crystallography

Birkbeck College, Malet Street

WC1E 7HX London, UK
The accurate prediction of the biochemical function of a protein is becoming increasingly important, given the unprecedented growth of both structural and sequence databanks. Consequently, computational methods are required to analyse such data in an automated manner to ensure genomes are annotated accurately. Protein structure prediction methods, for example, are capable of generating approximate structural models on a genome-wide scale. However, the detection of functionally important regions in such crude models, as well as structural genomics targets, remains an extremely important problem. The method described in the current study, MetSite, represents a fully automatic approach for the detection of metal-binding residue clusters applicable to protein models of moderate quality. The method involves using sequence profile information in combination with approximate structural data. Several neural network classifiers are shown to be able to distinguish metal sites from non-sites with a mean accuracy of $94.5 \%$.

The method was demonstrated to identify metal-binding sites correctly in LiveBench targets where no obvious metal-binding sequence motifs were detectable using InterPro. Accurate detection of metal sites was shown to be feasible for low-resolution predicted structures generated using mGenTHREADER where no side-chain information was available. High-scoring predictions were observed for a recently solved hypothetical protein from Haemophilus influenzae, indicating a putative metal-binding site.

(C) 2004 Elsevier Ltd. All rights reserved.

Keywords: functional site; structural genomics; PSSM; metal binding; model proteins ahead. Gene products from different species may exhibit similar biological function, but show little or no sequence similarity due to convergent evolution. Structural classification of protein domains such as $\mathrm{CATH}^{2}{ }^{2} \mathrm{SCOP}^{3}$ and $\mathrm{FSSP}^{4}$ reveals that members of the same structural family can span different functional classes. Furthermore, key active sites may be conserved despite there being little overall structural and sequence similarity. It is therefore clear that analysis of functional regions will allow the development of more reliable genome annotation and enhance our knowledge of the biological role of proteins at a cellular level.

Historically, structural insights have provided the most detailed information on biological function, highlighting, for example a specific catalytic mechanism or interactions with other molecules. An indepth analysis of key functional regions such as
Abbreviations used: ANN, artificial neural networks; ROC, receiver operating characteristic; SS, secondary structure; Solv, solvent accessibility; PSSM, positionspecific scoring matrix; DM, distance matrix; FPR, falsepositive rate; TPR, true-positive rate.

E-mail address of the corresponding author: dtj@cs.ucl.ac.uk 
those in enzyme active sites, metal-binding sites, and ligand-binding clefts, as well as interacting regions between proteins is likely to add significantly to the repertoire of tools currently available.

Several groups have developed atomic-level methods for analyzing site regions. ${ }^{5-8}$ The TESS method $^{6}$ generates templates for locating geometric patterns in atoms occupying the site regions. This requires the accurate placement of specific sidechain atoms for sensitive site recognition and may not be suitable for model proteins. Fetrow and Skolnick have developed the "Fuzzy Functional Form" representation of a functional region ${ }^{7}$ combining information from the literature with sequence and structural analysis. This method can be applied to lower-resolution structures; however, the site under investigation must have been fully characterized and information must be retrieved manually from several sources. Bagley and Altman have developed the FEATURE ${ }^{9}$ method to characterize well-defined functional sites on the basis of statistical descriptors derived from a set of site and non-site data. FEATURE uses the exact placement of side-chain atoms as well as incorporating secondary structural information but does not include directly conservation information for site residues. The method was applied to locate calcium-binding sites in a set of model structures ${ }^{10}$ and was shown to require high-resolution placement of atoms specific to the site region.

Sequence searching tools have now become routine in initial investigations of new protein and DNA sequences. Pairwise comparison methods such as BLAST are generally effective only where sequence identity is at least $30 \%$. PSI-BLAST ${ }^{11}$ improves sensitivity by using sequence profiles and an iterative search strategy. Sequence-signature based methods such as PROSITE, ${ }^{12}$ PRINTS, ${ }^{13}$ Pfam, ${ }^{14}$ and BLOCKS ${ }^{15}$ search for sequence patterns within a query sequence. Generally, the sequence motif is specific to a functional family and can be used to infer functional information. These resources have been combined in the InterPro database, providing access to over 3000 entries. ${ }^{16}$ However, each of these methods has limitations. PROSITE regular expressions are effective for short motifs but the method fails in identifying members of highly divergent super-families. In contrast, the PRINTS fingerprints are derived from multiple sequence alignments and are particularly suitable for sub-family distinctions but fail at short motif recognition. The hidden Markov models used by Pfam provide a sensitive tool for identifying highly divergent members of super-families but may be less appropriate for sub-family predictions.

Residues that are not local in sequence but local in structure may form site regions. Sequence-based approaches cannot encode directly the 3D spatial organization of functional residues or the atoms responsible for biochemical action in the folded protein. Methods that combine sequence information with structural data offer a powerful approach for determining important functional locations. Rinaldis et al. have mapped sequence profile to surface structure to identify similarities in site regions of $\mathrm{SH} 2$ and $\mathrm{SH} 3$ domains as well as Ploop nucleotide-binding pockets. ${ }^{17,18}$

Protein structures crystallized in the absence of small-molecule substrates or metal ions highlight an alternative need to identify such functional regions automatically. Sites may be occupied by molecules found in buffering solutions, such as $\mathrm{SO}_{4}^{2-}$, thereby preventing binding of other prosthetic groups. Structural models provide an even harder challenge, prosthetic group binding for such cases will obviously need to be predicted computationally. The correct placement of sidechain atoms is rare, even for very good structural models. Methods capable of locating and classifying sites in predicted structures are therefore likely to improve the quality of genomic fold recognition efforts. Detailed analysis has been presented on the specific atomic geometry of metal sites in proteins. Karlin et al. undertook a comprehensive survey of residue and atomic preferences of metal ion ligation. ${ }^{19}$ Metal ion binding was investigated by Gregory et al., ${ }^{20}$ by developing hydrophobicity contrast measures, again using specific atomic placement on a limited dataset.

The rapid growth of the PDB and metal-containing structures alongside the explosion of sequence information allows a new opportunity to characterize functional sites.

Here, we present a novel approach using artificial neural networks (ANN) to predict six commonly occurring metal ion sites: $\mathrm{Ca}^{2+}, \mathrm{Cu}^{2+}, \mathrm{Fe}^{3+}, \mathrm{Mg}^{2+}$, $\mathrm{Mn}^{2+}$ and $\mathrm{Zn}^{2}$. The method is designed to identify residues forming the metal-binding site in superfamilies by combining sequence profile and structural information. The motivation of the study has been the development of functional site predictors where only moderate-quality structural information is available. Metal-binding site prediction was benchmarked for a set of newly released crystal structures from the LiveBench project. ${ }^{21}$ Site detection was shown to be effective in structural models of these targets. We report a putative metal-binding site predicted in a structural genomics target with unknown function.

\section{Results}

\section{Datasets}

The training set was constructed by taking all protein chains interacting with the specified metal ions from the PDB and clustering at a $25 \%$ sequence identity; this resulted in 1018 sequence clusters. For the purposes of cross-validation, these chains were then grouped into 364 distinct SCOP super-families. The numbers of PDB chains, super-families and metal sites in the dataset are summarized in Table 1. 
Table 1. Summary of dataset used to develop the MetSite method

\begin{tabular}{lccc}
\hline $\begin{array}{l}\text { Metal ion } \\
\text { type }\end{array}$ & $\begin{array}{c}\text { No. PDB } \\
\text { chains }\end{array}$ & $\begin{array}{c}\text { No. SCOP } \\
\text { super-families }\end{array}$ & $\begin{array}{c}\text { No. metal } \\
\text { ions }\end{array}$ \\
\hline $\mathrm{Zn}^{2+}$ & 512 & 190 & 803 \\
$\mathrm{Ca}^{2+}$ & 443 & 128 & 819 \\
$\mathrm{Mg}^{2+}$ & 349 & 124 & 470 \\
$\mathrm{Mn}^{2+}$ & 168 & 49 & 253 \\
$\mathrm{Cu}^{2+}$ & 86 & 11 & 110 \\
$\mathrm{Fe}^{3+}$ & 70 & 18 & 83 \\
\hline
\end{tabular}

\section{Feature analysis}

In order to determine the key features that allow effective discrimination of metal sites from nonmetal sites, fivefold cross-validation experiments were performed using only a subset of the site features (see Materials and Methods). During benchmarking, we ensure that no two protein chains occur within the same SCOP super-family between training and testing sets.

The classification results for individual feature sub-sets are illustrated in the form of receiver operating characteristic (ROC) plots (Figure 1). It is clear that structural information alone is not sufficient for sensitive classification, although it tends to improve marginally on the classification results using only position-specific scoring matrix (PSSM) scores of site residues. This highlights the important contribution of residue conservation in metal-binding residues and indicates a clear functional relevance.

On average, inclusion of PSSM scores together with secondary structure, site residue distances and solvent accessibility resulted in a $94.5 \%$ Q2 accuracy with a true-positive rate (TPR) of $39.2 \%$ at a $5 \%$ false-positive rate (FPR) threshold (Materials and Methods). Classification was marginally worse when training was performed using only the PSSM scores of site residues (TPR of 36.2\%). For comparison, training was performed using PSSM scores for residues local in primary sequence as opposed to local in structure, this resulted in a TPR of $30.5 \%$. Finally, classification using only the secondary structure assignments gave an average TPR of only $13.7 \%$. The average Wilcoxon statistic, which is a measure of the area under the ROC curve, over all classifiers was calculated to be $81.1 \%$ (where 100\% represents perfect classification).
In order to assess the significance of the neural network classification results, we compared the cross-validated predictions to a simple baseline prediction method using PSI-BLAST PSSM log likelihood scores. Metal-binding prediction was performed specifically for those residue types known to be more frequently occurring in the target metal sites. For example, in the case of calcium sites, the PSSM log likelihoods for Asp and Glu residues were extracted. Overall, we found that only $6.2 \%$ of metal-binding residues were predicted correctly using this approach at a 5\% FPR threshold. Table 2 shows the cross-validation classification results for the naive baseline and the full method on all sequence clusters.

\section{Site-based detection}

We find that a large proportion of patterns identified as sites under the above definition are not retrieved correctly during cross-validation under the allowable 5\% FPR. The encoding scheme, used in the training of MetSite produces many more site patterns than actual sites (Materials and Methods). For example, the 405 calcium sites produce 3529 site patterns, due to each residue in the vicinity of a metal-binding site being labelled as a site residue. However, each of these residues actually belongs to a unique site region in the crystal structure. The MetSite method was therefore assessed by its ability to predict unique site regions. This was achieved by summing the neural network outputs for individual residues within a $7 \AA$ radius giving an overall score for the site. This resulted in the correct prediction of $60 \%$ of all metal sites in all super-families within the top-ranking MetSite predictions.

\section{Site prediction in SCOP super-families}

The cross-validated classification performance was investigated for each of the most highly populated SCOP super-family clusters. The topranking site predictions for these over-represented super-family members are presented in Table 3 and clearly indicate significantly better metal-site predictions. Within the calcium-containing protein chains, the EF-hand-like domains made up the most prevalent cluster consisting of 71 unique sites, of these $61(85.9 \%)$ were predicted correctly with a

Table 2. Overall cross-validation classification results at 5\% false-positive rate (FPR)

\begin{tabular}{|c|c|c|c|c|}
\hline Metal ion & Q2 accuracy (\%) & TPR (\%) & Wilcoxon & Naïve TPR (\%) \\
\hline $\mathrm{Ca}^{2+}$ & 93.9 & 30.4 & 79.9 & 5.0 \\
\hline $\mathrm{Cu}^{2+}$ & 94.9 & 36.2 & 85.6 & 2.7 \\
\hline $\mathrm{Fe}^{3+}$ & 94.9 & 48.8 & 84.0 & 8.9 \\
\hline $\mathrm{Mg}^{2+}$ & 94.2 & 32.4 & 73.8 & 6.8 \\
\hline $\mathrm{Mn}^{2+}$ & 94.7 & 38.8 & 80.8 & 8.0 \\
\hline $\mathrm{Zn}^{2+}$ & 94.6 & 47.8 & 82.2 & 6.0 \\
\hline
\end{tabular}

The true-positive rate (TPR), Q2 accuracy and Wilcoxon statistic are defined in Materials and Methods. The naïve TPR represents a baseline using simple residue conservation score. 

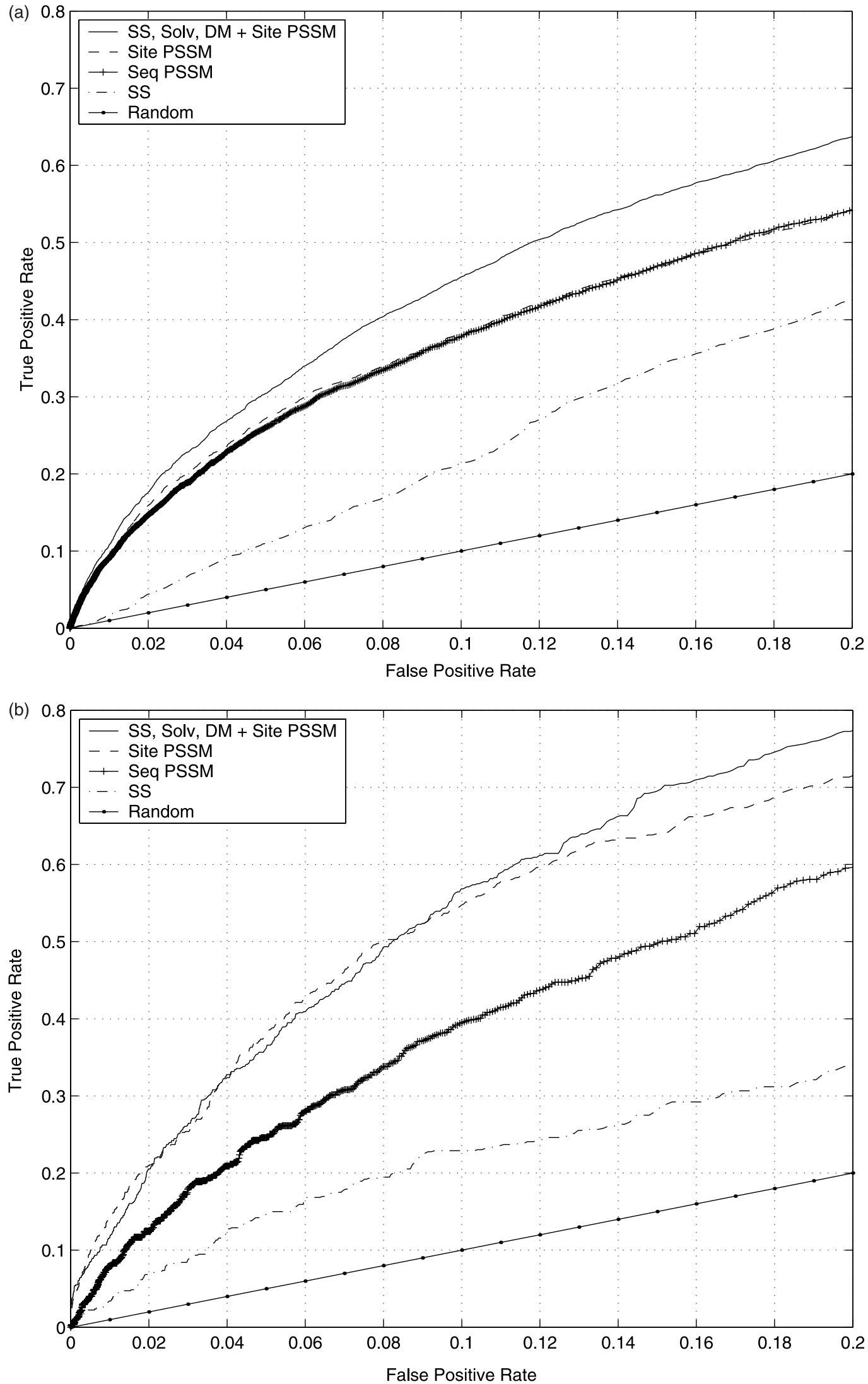

Figure 1 (legend on p. 312) 


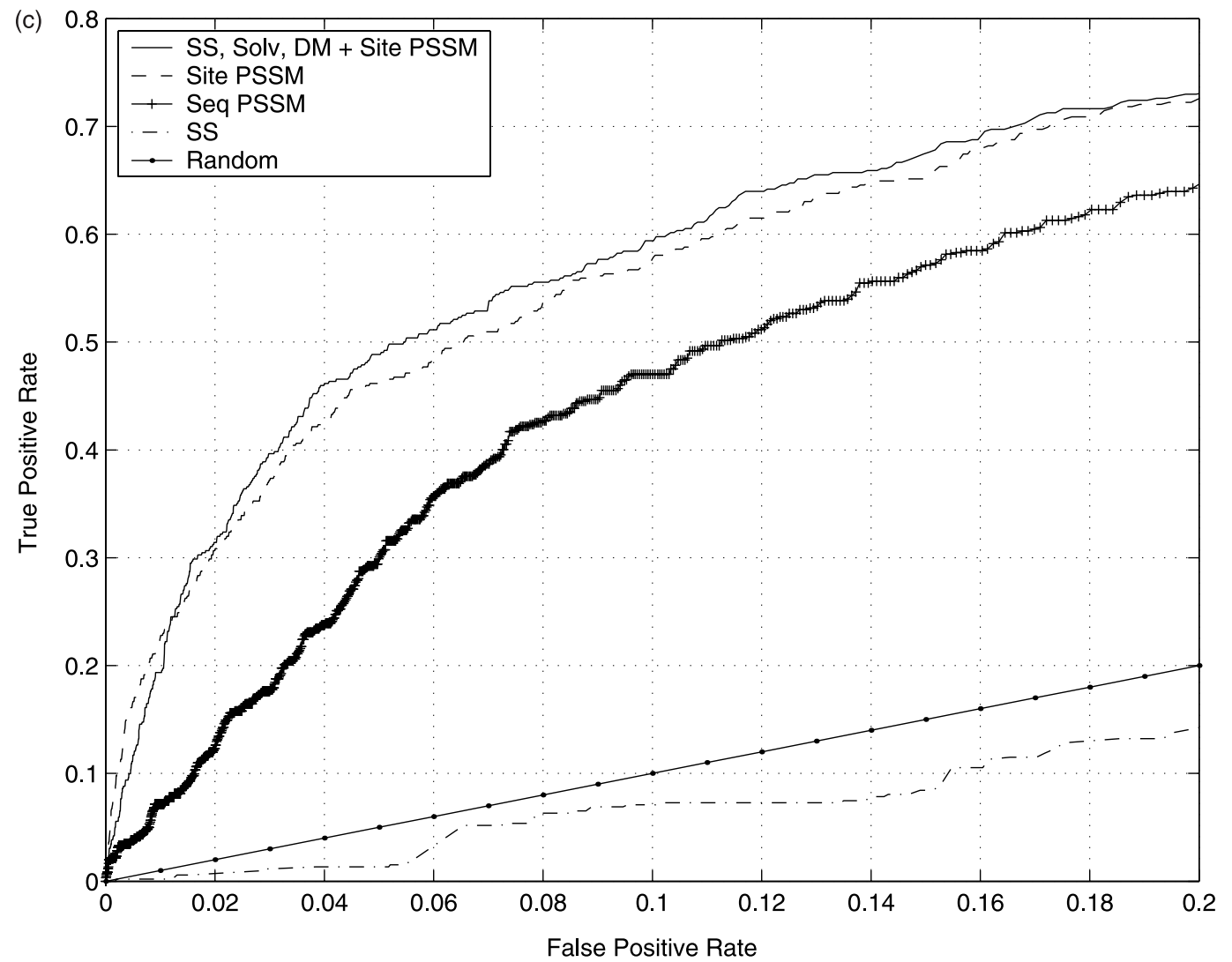

(d) 0.8 - SS, Solv, DM and Site PSSM

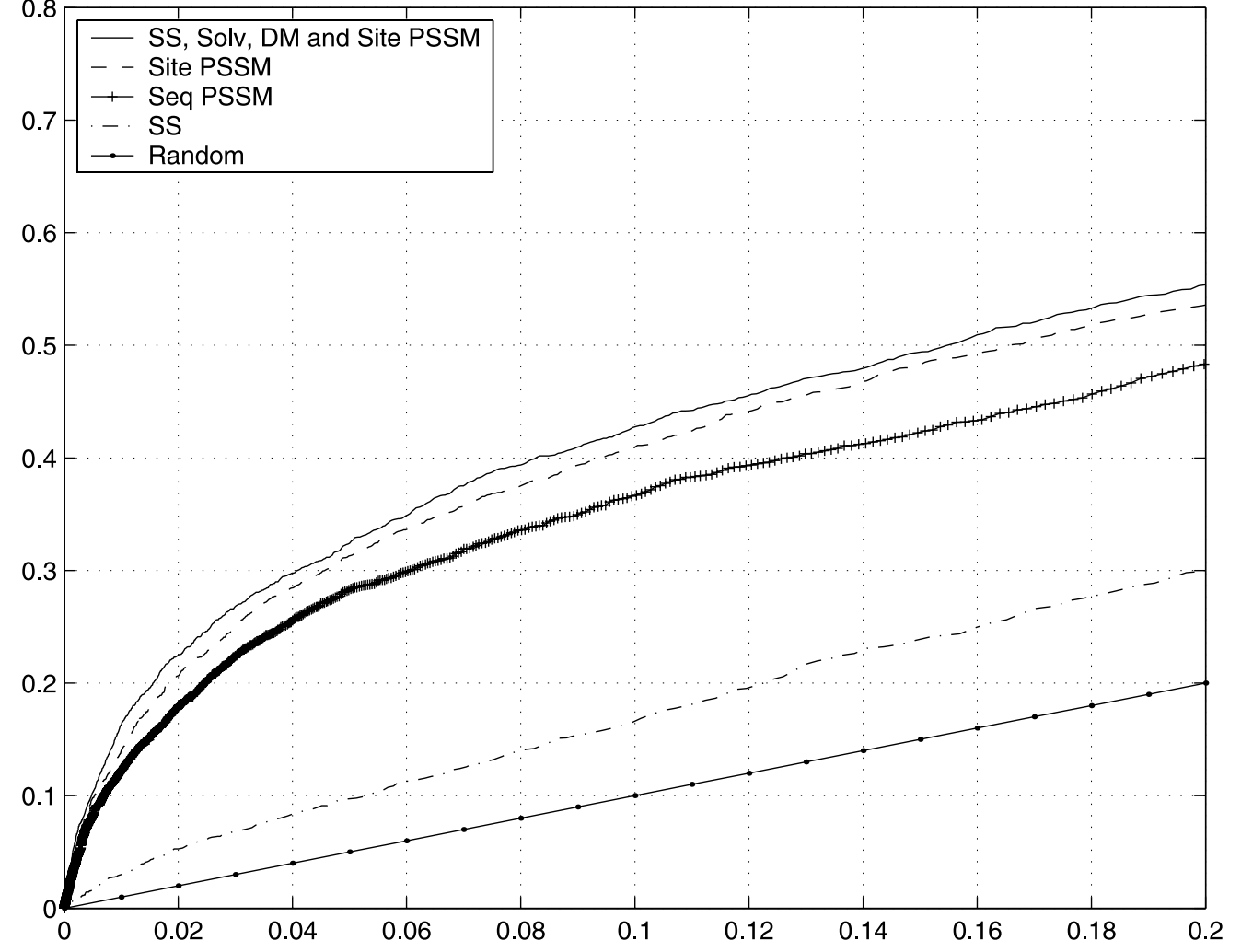

Figure 1 (legend on p. 312) 

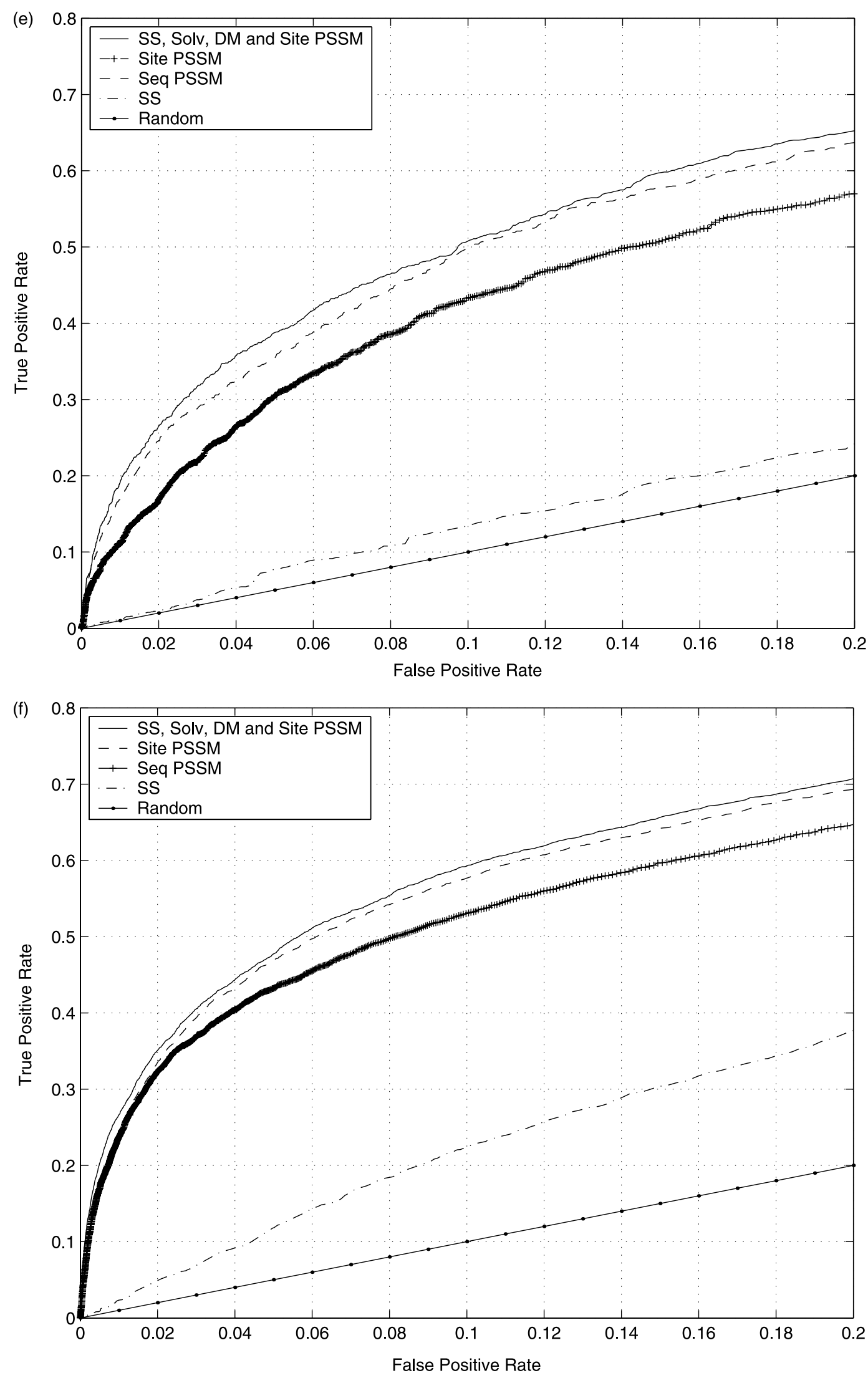

Figure 1. Receiver operating characteristic (ROC) curves to assess classification performance using various feature sub-sets for each metal site type; (a) $\mathrm{Ca}^{2+}$, (b) $\mathrm{Cu}^{2+}$, (c) $\mathrm{Fe}^{3+}$, (d) $\mathrm{Mg}^{2+}$, (e) $\mathrm{Mn}^{2+}$ and (f) $\mathrm{Zn}^{2+}$. Fivefold cross-validation was repeated for each of the metal site types using several combinations of the features. Features investigated in this study were: secondary structure (SS); solvent accessibility (Solv); position-specific scoring matrix (PSSM); and distance matrix (DM), defined as distances between site $C^{\beta}$ atoms $\left(C^{\alpha}\right.$ for glycine). The classification was also assessed for residues local in primary sequence (Seq PSSM). 
Table 3. Site-based predictions in over represented SCOP super-families

\begin{tabular}{|c|c|c|c|c|}
\hline SCOP super-family & $\begin{array}{l}\text { Super-family } \\
\text { representative }\end{array}$ & Total sites & Site sensitivity (\%) & Site selectivity (\%) \\
\hline \multicolumn{5}{|l|}{ A. Calcium } \\
\hline EF hand & 1alvB & 71 & 85.9 & 73.5 \\
\hline Phospholipase A2 & $1 \mathrm{~g} 4 \mathrm{iA}$ & 6 & 100 & 40.0 \\
\hline C-type lectin & & 6 & 75.0 & 54.5 \\
\hline Concanavalin A & & 15 & 60.0 & 23.0 \\
\hline \multicolumn{5}{|l|}{ B. Zinc } \\
\hline $\mathrm{C} 2 \mathrm{H} 2$ and $\mathrm{C} 2 \mathrm{HC}$ zinc fingers & $1 \mathrm{f} 2 \mathrm{iK}$ & 24 & 72.7 & 57.1 \\
\hline Metalloproteases ("zincins") & 1ast0 & 17 & 58.8 & 26.3 \\
\hline Glucocorticoid & $1 a 6 y B$ & 20 & 80.0 & 55.2 \\
\hline NAD(P)-binding Rossmann-fold & $1 \mathrm{e} 31 \mathrm{~A}$ & 6 & 83.3 & 38.5 \\
\hline Zn-dependent exopeptidases & $1 \mathrm{cg} 2 \mathrm{~B}$ & 12 & 91.6 & 40.7 \\
\hline \multicolumn{5}{|l|}{ C. Magnesium } \\
\hline P-loop hydrolase & $1 \mathrm{a} 820$ & 35 & 88.6 & 32.0 \\
\hline ATPase domain & 1byqA & 5 & 90.0 & 45.5 \\
\hline Phosphoenolpyruvate/pyruvate & 1dxeA & 4 & 83.3 & 27.7 \\
\hline Protein kinase & $1 \mathrm{blxA}$ & 9 & 91.6 & 30.8 \\
\hline \multicolumn{5}{|l|}{ D. Copper } \\
\hline Cupredoxin & $1 \mathrm{a} 4 \mathrm{aA}$ & 32 & 62.5 & 44.4 \\
\hline \multicolumn{5}{|l|}{ E. Manganese } \\
\hline $\mathrm{Fe} / \mathrm{Mn} \mathrm{SOD}$ & $1 g v 3 A$ & 1 & 100 & 50.0 \\
\hline \multicolumn{5}{|l|}{ F. Iron } \\
\hline Ferritin & $1 \mathrm{~b} 71 \mathrm{~A}$ & 15 & 100 & 32.0 \\
\hline Rubredoxin & $1 \mathrm{~b} 13 \mathrm{~A}$ & 4 & 100 & 33.3 \\
\hline $\mathrm{Fe} / \mathrm{Mn} \mathrm{SOD}$ & $1 g v 3 \mathrm{~A}$ & 1 & 100 & 33 \\
\hline Clavaminate synthase-like & $1 \mathrm{bk00}$ & 3 & 66.7 & 10 \\
\hline
\end{tabular}

selectivity of $73.5 \%$ (see Materials and Methods). Similarly, site detection was much more accurate for all structures in the over-represented families where metal binding shows a clear functional relevance. Overall site sensitivity of metal sites for these clusters was $85 \%$ with a selectivity of $39 \%$. Given that the neural network is trained such that no two proteins in training/testing fall within the same SCOP super-family, these results indicate that MetSite has effectively generalized site characteristics.

\section{Confidence and distinction between metal sites}

It is essential that results have accurate confidence values assigned to them to permit the statistical significance of any finding to be assessed. Also, in the case where several networks produce high scores, we need to predict the most likely type of metal-binding site. In order to accomplish this, we determine the log likelihood ratio for correct prediction against network score for each of the networks (Materials and Methods).

The log likelihood ratio of correct prediction against network output score is given in Figure 2. This allows us to rank the confidence of the different prediction methods as a function of network score, thereby allowing direct comparisons to be made between the different classifiers. In practice, the classifier producing the highest log likelihood score would be taken as the site prediction. However, it is plausible that more than one classifier may produce a high likelihood score (for instance in the $\mathrm{Cu}(\mathrm{II})$ / $\mathrm{Zn}$ superoxide dismutase active site). For reference, at a log likelihood score of 2 there are 100 correct predictions for every false positive, indicating a high level of confidence (Figure 2).

\section{Site prediction in LiveBench targets}

The LiveBench project is a continuous structure prediction assessment for newly determined structures, including targets from the various structural genomics projects. These target structures are of particular interest, as they show no significant sequence similarity to any other known protein structures. MetSite was used to scan 172 protein chains from LiveBench-8. Of these, 24 chains contained occurrences of target metal ions in the crystal structure. The top-ranking MetSite predictions identified the true metal binding region correctly in 19/24 (71.2\%) of the crystal structures.

\section{Identification of POP2 metal-binding site}

The RNase domain of the yeast POP2 protein (1uocA) was predicted to bind $\mathrm{Mn}^{2+}$ with high confidence in a site region centered around Ser44. The predicted site was devoid of any prosthetic group, although the protein did contain several calcium ions at different site regions. An inspection of the literature revealed the active-site Ser44 of this protein is in fact involved in $\mathrm{Mn} / \mathrm{Mg}$ binding ${ }^{22}$ and makes up part of the active-site region. The authors speculate that POP2 binds only a single metal ion instead of the two metal ions observed for the DNases, resulting in a different reaction mechanism. This is consistent with the MetSite predictions, 


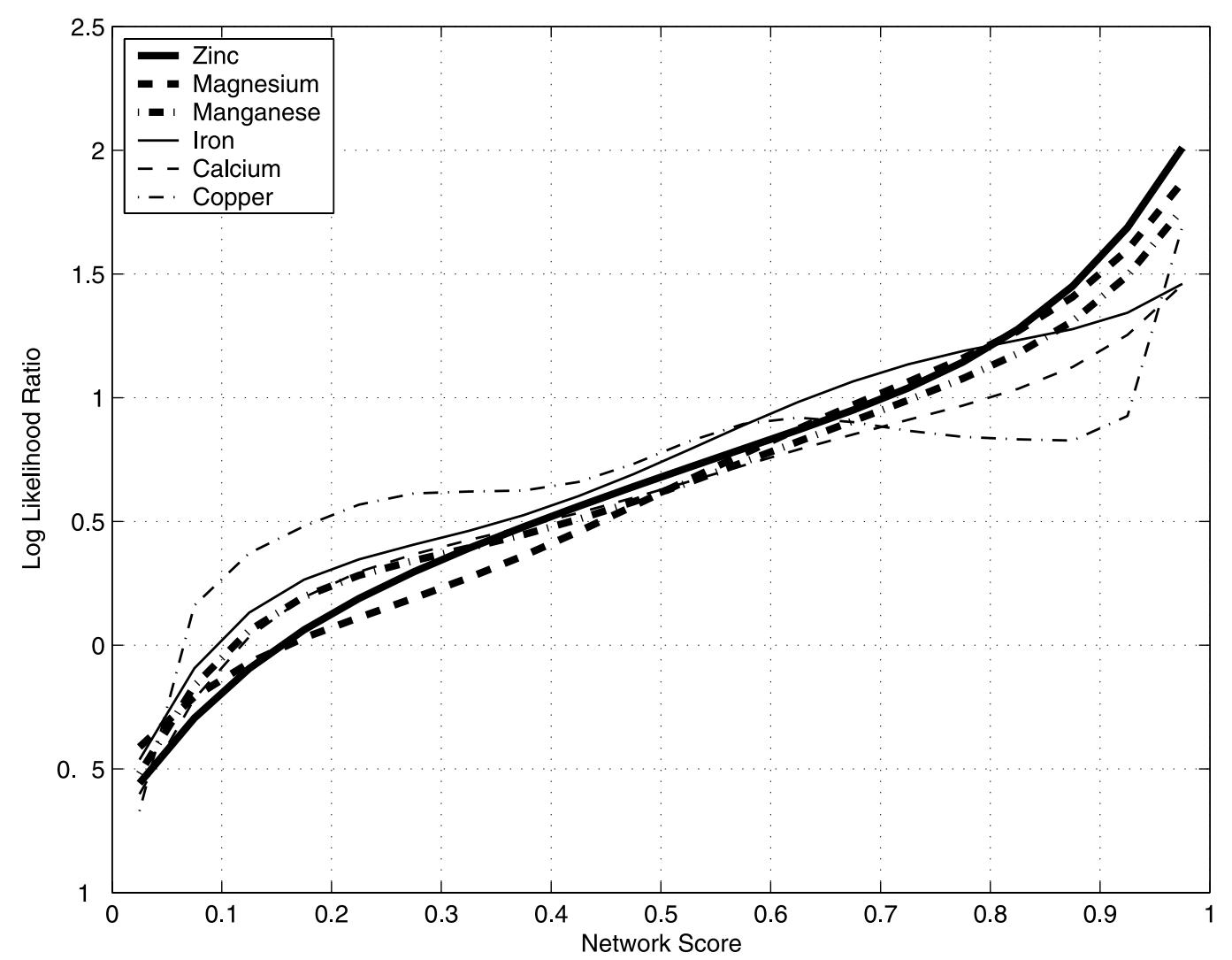

Figure 2. Likelihood ratio plots for the different metal site classifiers used to assess the statistical significance of the raw neural network outputs.

as only a single strong hit was observed, even though no metal is present in the active site of the crystal structure.

\section{Modelling of LiveBench targets}

Using the mGenTHREADER fold recognition method, ${ }^{23,24}$ we assessed the ability of MetSite to locate metal-binding regions in structural models. It is possible to obtain good-quality models using fold recognition techniques such as mGenTHREADER even when there is scant sequence identity between target and template. Models derived from mGenTHREADER alignments do not contain coordinates of side-chain atoms but rather the predicted location of the target protein's backbone; nevertheless, such models are suitable for scanning with MetSite, which requires only the approximate locations of residues.

All proteins within the LiveBench dataset were screened against a non-redundant fold library using the distributed version of mGenTHREADER. ${ }^{25,26}$ In order to remove trivial cases where binding sites could be deduced from simple homology searches, we focused our analysis on target/template pairs that showed $<30 \%$ sequence identity. In addition, we ensured that template structures did not contain any obvious metal-binding sites. The secondary structure of each target protein was predicted using PSIPRED. ${ }^{27}$

The mGenTHREADER predictions of the 24 metal-containing LiveBench targets produced 15 models with MaxSub ${ }^{28}$ scores $>0$ (where 100 indicates a perfect structural prediction) in the top-ranking hits (Table 4).

\section{Predicting sites in fold recognition models}

Of the 15 metal-containing LiveBench model structures, MetSite identified the metal-binding region correctly in eight targets (53\%) within the top site predictions (Table 4). Figure 3 illustrates the MetSite predictions for the native and model structures of zinc-containing phosphonoacetate hydrolase (1ei6A) and the catalytic domain of $N$ acetylmuramoyal-L-alanine amidase (1jwqA). An InterPro search of these protein sequences did not reveal any obvious metal-binding motif. The topranking MetSite predictions indicate that all residues chelating the zinc ion in both proteins have been identified correctly and cluster around the actual metal site with no false positive hits. The topranking $m$ GenTHREADER result for 1ei6A predicts a hit with the template structure human arylsulfatase (1fsu0) with an $E$-value of $3 \times 10^{-4}$, although the sequence identity between these proteins is only $9.1 \%$. Figure 4(c) illustrates the top-ranking MetSite predictions for the 1ei6A model structure; all the top hits for 1ei6A were located in the vicinity of the actual $\mathrm{Zn}^{2+}$ site, even though only a small portion of the structure was modelled correctly. The MetSite scan of the 1jwqA model produced only a single hit 
Table 4. Top ranking mGenTHREADER predictions for metal-containing LiveBench-8 targets

\begin{tabular}{|c|c|c|c|c|}
\hline LiveBench target & Template structure & Sequence identity (\%) & MaxSub score & $E$-value \\
\hline $104 t A$ & $11 r 5 \mathrm{~A} 0$ & 15.0 & 66 & 0.045 \\
\hline $1 \mathrm{~m} 3 \mathrm{uA}$ & $1 \mathrm{mumA} 0$ & 13.3 & 42 & $2 e^{-4}$ \\
\hline $10 y 0 A$ & $1 \mathrm{mumA} 0$ & 13.2 & 41 & $4 e^{-4}$ \\
\hline 1iujA & $11 \mathrm{q} 9 \mathrm{~A} 0$ & 16.0 & 54 & 0.04 \\
\hline 1qvjA & $1 \mathrm{kt} 9 \mathrm{~A} 0$ & 21.2 & 20 & 0.049 \\
\hline $1 \mathrm{mzbA}$ & $1 \mathrm{gbjA0}$ & 20.0 & 38 & 0.159 \\
\hline 1rifA & 1qvaA0 & 15.5 & 36 & 0.004 \\
\hline $1 \mathrm{uf} 3 \mathrm{~A}$ & 1nnwA0 & 13.2 & 38 & 0.003 \\
\hline 1ei6A & $1 \mathrm{fsu} 00$ & 9.1 & 25 & $3 e^{-4}$ \\
\hline 1uocA & $1 f \times x A 0$ & 10.4 & 37 & 0.013 \\
\hline 1qv9A & $1 \mathrm{f} x \mathrm{xA} 0$ & 11.7 & 16 & 0.03 \\
\hline 1jwqA & $1 \mathrm{di} 0 \mathrm{~A} 0$ & 10.1 & 29 & 0.079 \\
\hline $104 z A$ & 1dypA0 & 15.9 & 42 & 0.004 \\
\hline $104 y A$ & 1 dypA0 & 15.9 & 39 & 0.003 \\
\hline 1uetA & $1 \mathrm{fa} 0 \mathrm{~B} 0$ & 10.1 & 25 & $2 e^{-4}$ \\
\hline
\end{tabular}

The MaxSub score was taken at 3.5 cut-off and E-value corresponds to mGenTHREADER confidence. The bold targets represent cases for which metal sites were predicted correctly in the model structure.

with high confidence, corresponding to a His residue involved in zinc binding in the native structure. Figure 3(c) and (d) illustrates that the structural predictions may be regarded as only moderate quality when compared to the native LiveBench targets. Indeed, both modelled proteins gave MaxSub scores below 30. Nonetheless, residues involved in metal binding were identified correctly for both predicted protein structures.

\section{Site prediction in a hypothetical protein}

Functionally unannotated structures are obviously of particular interest and require accurate methods to identify correctly the location and identity of functional site regions. The LiveBench8 set contained 42 structures with unknown function annotations. Analysis of the MetSite predictions for these structural genomics targets revealed high-scoring hits for the Haemophilus influenzae hypothetical protein HI0817 (PDB code 1izmA). A BLAST sequence comparison of this target sequence revealed only five hits with an $E$-value of less than $10^{-3}$, all of which were also hypothetical proteins.

The best mGenTHREADER prediction for this target produced a MaxSub score of only 13 against the NMR structure of apolipophorin-III (PDB 1eq1A). ${ }^{29}$ This lipid-binding protein is classified as a five-helix bundle belonging to the apolipophorinIII super-family according to SCOP. However, the sequence identity between HI0817 and apolipophorin-III is only $9.8 \%$, and the mGenTHREADER $E$-value for the structural alignment is 0.068, suggesting that these structures are unlikely to be related functionally.

The $\mathrm{Fe}^{3+}$ classifier produced a strong hit against HI0817 with the top five ranking predictions producing network output scores $>0.7$. In order to visualize the predictions, the network outputs were mapped onto the protein structure (Materials and Methods). Figure 4 demonstrates all of these hits to be clustered around the same site region corresponding to residues Gly26, Gln105, Asp101,
Asn104, and Glu23. The overall log likelihood ratio of this site region was calculated to be 5.94, a strong indication of a putative $\mathrm{Fe}^{3+}$ binding site.

\section{Discussion}

We have developed MetSite, a set of artificial neural network classifiers that have been optimized to locate metal-binding regions in protein structures and were shown to predict metal sites in modelled protein structures from LiveBench accurately. Position-specific scoring matrices for metal-binding residues, as well as residues forming the second coordination shell interactions, were shown to be sufficient for discriminating metal ion sites from non-sites. Secondary structure, solvent accessibility and distance matrices of site residues improve classification performance. The improved classification results obtained by extracting profile scores from residues forming the $3 \mathrm{D}$ site as opposed to residues local in primary sequence illustrates the importance of implicit spatial encoding. We restrict ourselves to only the approximate location of site residues, allowing sites to be identified from homology models where only the approximate backbone conformation is available. An important aspect of the benchmarking is to ensure no two members of a SCOP super-family are present in both training and testing sets, thereby mimicking site detection in proteins belonging to new superfamilies. Overall accuracy of site/non-site prediction is high $(94.2 \%)$ at a low false-positive rate of $5 \%$, the method is reasonably sensitive at this threshold, retrieving $60 \%$ of all sites correctly.

Analyses of the most prominent SCOP superfamilies for each of the metal types investigated reveal that the method is much more sensitive and selective at identifying metal-binding site residues in these structures. On average, we predict over $84 \%$ of metal sites in these structures correctly with a selectivity of $39 \%$, significantly better performance than over the complete dataset. This is a clear 

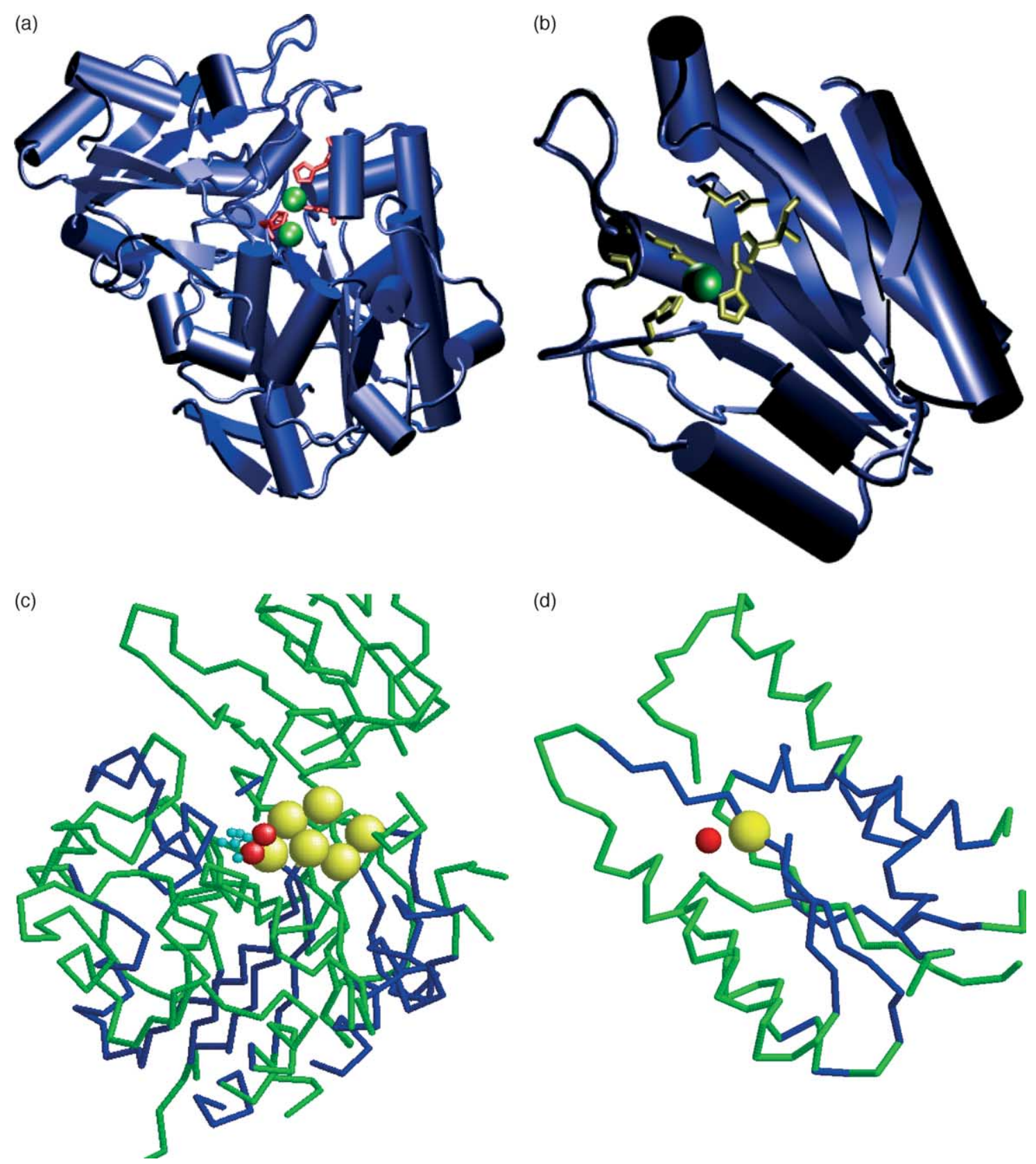

Figure 3. MetSite prediction of LiveBench targets. Crystal structures of (a) N-acetylmuramoyl-L-alanine amidase (1ei6A) and (b) phosphonoacetate hydrolase (1jwqA). The mGenTHREADER predicted structures shown in (c) and (d) correspond to (a) and (b), respectively. MetSite predictions are coloured yellow.

indication that many of the metal sites present in the dataset are likely to be the result of artefacts of crystallization and, as such, do not exhibit the sequence/structure characteristics that may indicate a biological significance. Sites may be occupied by different ion types; for example, the calciumbinding protein 2pal (calmodulin) is crystallized with a manganese ion occupying the actual calcium site. Detection and identification is complicated further by the fact that many active-site regions contain more than a single type of metal, such as those found in superoxide dismutases. However, a robust approach based on likelihood estimates enables site prediction to be assigned a confidence value, thereby allowing distinction between different site types.

Validation of MetSite on targets from LiveBench8 demonstrates the generalization abilities of the classifiers. These newly released structures do not share any significant sequence similarities to proteins in the PDB; nonetheless MetSite identified the true metal-binding site correctly for over $71 \%$ of these difficult targets within the top ranking predictions. Accurate predictions were observed also for the active-site $\mathrm{Mn}^{2+}$ of the RNase domain of the yeast POP2 protein (1uocA), even though the site was not crystallized with the metal ion present. Manual inspection of the literature revealed that the 

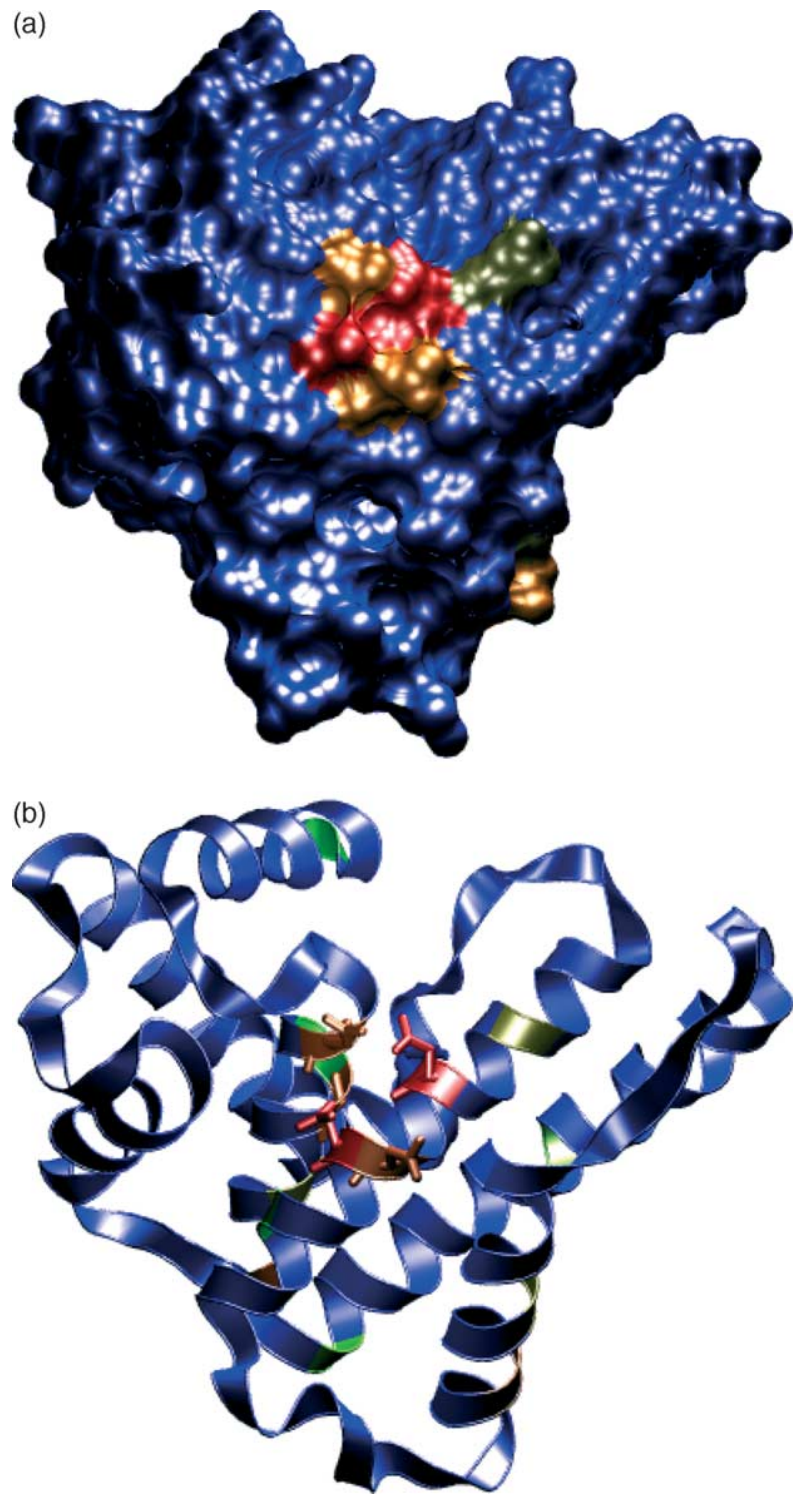

Figure 4. MetSite predictions for the crystal structure of structural genomics target HI0817 from H. influenzae (PDB code 1izmA). (a) Surface representation and (b) ribbon representation. The $\mathrm{Fe}^{3+}$ neural network scores have been mapped to the temperature factor column in the PDB file, high-scoring predictions indicating a putative metal-binding site are coloured red.

high-scoring hits for this target were indeed biologically important metal sites. Closer inspection of two zinc-containing targets (1ei6A and 1jwqA), for which no obvious metal site was detected using the sequence motif searching methods in InterPro, revealed all chelating residues were correctly identified with no false-positives.

The MetSite results for structural genomics targets within the LiveBench set revealed a highscoring hit for the hypothetical protein HI0817 from $H$. influenzae, indicating a putative $\mathrm{Fe}^{3+}$-binding site. Sequence comparison as well as an InterPro scan of this target sequence did not detect any obvious functional site.
Metal site detection was shown to be possible in the current study for mGenTHREADER-predicted structures of the LiveBench set where only approximate backbone position is available. Metal-site residues were identified correctly in 8/15 targets for model structures with MaxSub score $>0$ using only relative backbone coordinates.

The advantages of the machine learning approach presented here are twofold. Firstly, we do not rely upon the specific placement of sidechain atoms that are the most prominent ligating donors in the metal sites investigated. We restrict our inputs for each residue to the position of their $\mathrm{C}^{\beta}$ ( $\mathrm{C}^{\alpha}$ for glycine) atoms and therefore are more tolerant to errors in poor-quality structures as well as homology models. The second advantage is the speed of the classification, a single protein can be processed within 0.5 seconds (using a $1.5 \mathrm{GHz}$ AMD running Linux) given the PSI-BLAST PSSM, enabling genome-wide classification. Furthermore, a robust approach based on likelihood enables site prediction to be assigned a confidence value.

Functional site predictions in modelled structures are likely to complement fold-recognition methods; the correct spatial clustering of functionally important residues could be used as a measure of structure prediction quality, and efforts are underway to determine how MetSite may be extended to improve structural predictions.

Future directions will be to apply the technique developed for MetSite to larger functional site regions and application of the method to structural models that have been generated across genomes for the Genomic Threading Database. ${ }^{25,26}$ The use of functional site predictions to improve ranking of modelled structures will be investigated.

\section{Materials and Methods}

The PSI-BLAST score matrices were derived by performing three iterations of PSI-BLAST against a nonredundant database for all the unique chains across all datasets. We defined metal site seed residues as those residues with main-chain atoms within $7 \AA$ of a metal ion, $N$ closest neighbouring residues to these seeds were marked as seed neighbours (Figure 5).

For each marked residue, several features were calculated. These included the 20 scores taken from the PSI-BLAST PSSM, secondary structure state (reduced to helix, sheet or coil), solvent accessibility from $\operatorname{DSSP}^{30}$ and, finally, the inter-atomic distances between the $C^{\beta}\left(C^{\alpha}\right.$ for glycine) atoms for the site residues. Thus for each site, consisting of $n$ residues (seed $+(n-1)$ neighbours), we defined 20n PSI-BLAST profile scores, $3 n$ secondary structure states, $n$ solvent-accessibility scores and an inter-atomic distance matrix between the $n$ residues $(n(n-1) / 2)$.

The metal ion environment that forms the second coordination shell as well as regions further away from the ligand residues play a crucial role in site selectivity; however, in the context of the site description used here, increasing the number of residues used to compose a site pattern results in over-fitting problems during training. 


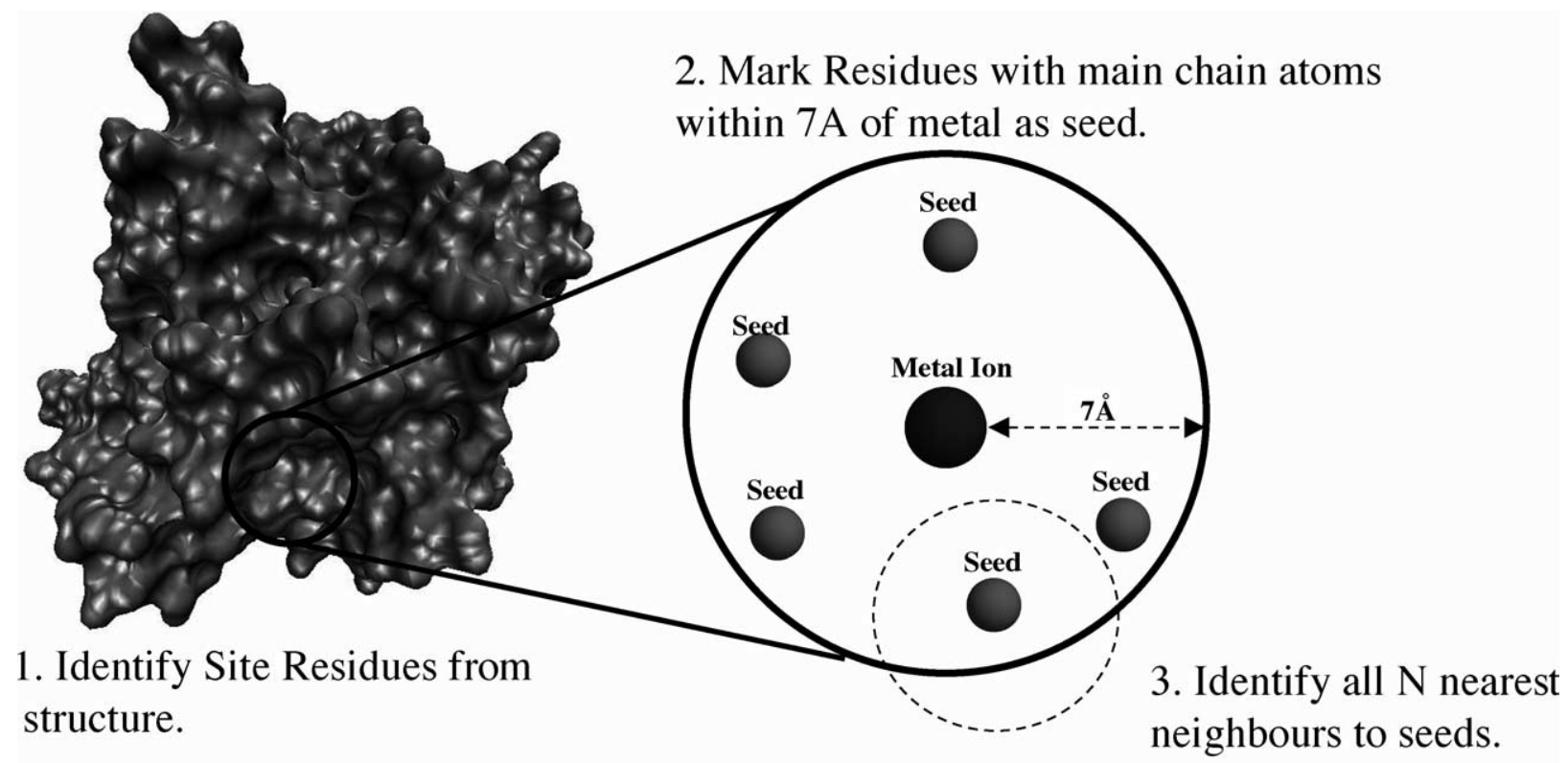

\section{Extract PSSM scores, DSSP assignments and calculate DM} for all marked residues.

\begin{tabular}{|c|c|c|c|c|c|c|c|c|c|c|c|c|c|c|c|c|c|c|c|c|c|}
\hline & & A & R & $\mathrm{N}$ & D & C & Q & E & G & $\mathrm{H}$ & I & L & K & M & $\mathrm{F}$ & P & S & $\mathrm{T}$ & W & $Y$ & V \\
\hline & & -1 & -4 & -3 & -4 & 10 & -4 & -4 & -3 & -4 & -2 & -2 & -4 & -2 & -3 & -4 & -1 & -1 & -3 & -3 & -1 \\
\hline 2 & G & 0 & -3 & -1 & -2 & -3 & -2 & -3 & 6 & -3 & -5 & -4 & -2 & -3 & -4 & -3 & -1 & -2 & -3 & -4 & -4 \\
\hline 3 & $\mathrm{~V}$ & 2 & -1 & -2 & -3 & -2 & -1 & -2 & -2 & -3 & 1 & -1 & 2 & -1 & -2 & -2 & -1 & -1 & -4 & -2 & 3 \\
\hline 4 & P & -1 & -3 & -3 & -2 & -4 & -2 & -2 & -3 & -3 & -3 & -4 & -2 & -3 & -4 & 8 & -1 & -2 & -4 & -4 & -3 \\
\hline 5 & A & 2 & -1 & -1 & -1 & -1 & 3 & 0 & -1 & -1 & 2 & -1 & -1 & 0 & -2 & -1 & 0 & -1 & -2 & -1 & 0 \\
\hline 6 & I & -1 & -2 & -2 & -2 & -1 & -2 & -2 & -2 & -2 & 3 & 0 & -2 & 0 & 4 & -2 & -2 & -1 & -1 & 0 & 3 \\
\hline 7 & $Q$ & -2 & 3 & -1 & -1 & -4 & 5 & 3 & -3 & 0 & -4 & -3 & 1 & -2 & -4 & -2 & -1 & -1 & -3 & -2 & -3 \\
\hline 8 & $\mathrm{P}$ & -1 & -2 & -2 & -1 & -4 & -1 & 2 & -3 & -2 & -4 & -4 & -1 & -3 & -4 & 7 & -1 & -2 & -4 & -3 & -3 \\
\hline 9 & $\mathrm{~V}$ & -2 & -1 & 2 & -2 & -3 & -1 & -2 & -3 & -2 & -1 & -2 & 2 & -2 & 3 & 4 & -1 & -1 & -3 & -1 & 1 \\
\hline 10 & L & -1 & -1 & -2 & -3 & -2 & -1 & -2 & -3 & -2 & -1 & 3 & 2 & 0 & 4 & -3 & 1 & -1 & -2 & 0 & -1 \\
\hline 11 & $S$ & -1 & 2 & -1 & -1 & 5 & 0 & 2 & -2 & -2 & -3 & -3 & 0 & -2 & -3 & -2 & 3 & 0 & -3 & -3 & -2 \\
\hline 12 & G & 1 & -2 & -2 & -1 & -3 & -1 & 2 & 2 & -2 & -3 & -3 & -1 & -3 & -4 & 5 & -1 & -2 & -4 & -3 & -3 \\
\hline
\end{tabular}

Figure 5. Definition of site patterns. (DM, distance matrix).

We therefore restricted $N$ to 10 , which equates to 285 features and was shown to allow good generalization.

Classification was also performed using PSSM scores for residues proximal in protein sequence. As for the sitebased method described above, all residues within $7 \AA$ of a metal ion are marked as seed residues; however, the neighbouring residues now become those local in sequence.

\section{Pre-processing of site data}

Prior to neural network training, the inputs applied to each of the residues were rescaled in the range $[0,1]$ by using the standard sigmoid logistic function:

$$
y=\frac{1}{1+\exp ^{(-a x)}}
$$

where $x$ is the raw input value, $a$ is an arbitrary constant and $y$ is the rescaled value.

For each metal type classifier, a three-layer, singleoutput, feed-forward neural network was created and trained using the neural network toolbox in Matlab (H. Demuth and M. Beale, unpublished). The number of units in the hidden layer is an important factor in preventing over-fitting on the training data, especially in cases where limited training examples are available. It was found that 25 nodes in the hidden layer, trained using resilient back-propagation ${ }^{31}$ and early stopping, resulted in good generalization on the testing sets. For each training run, $10 \%$ of the training data was used as a validation set to evaluate the performance of the network during training and prevent over-fitting.

We screened all PDB chains within the dataset for each metal ion in turn. All residues outside the interacting range of a given metal ion were taken as negative sites. The 1018 sequence clusters were grouped at the SCOP super-family level, resulting in 364 clusters. We randomly split these SCOP clusters into five groups and carried out fivefold cross-validation. This rigorous validation approach ensures that no two site patterns between training and test sets had any similarity at the superfamily level, therefore mimicking site detection in new super-families. This allows the generalization characteristics of each MetSite classifier to be assessed in an extremely robust manner.

The results from the complete cross-validation test for each of the metal site datasets were pooled to calculate prediction accuracy, defined as the total number of true positive and true negatives over all patterns $(Q 2)$. We assess TPR and FPR as defined below. The nonparametric Wilcoxon statistic, representing the area under the ROC curve, is a robust measure for comparing classifiers and was calculated to determine the 
significance between classifications of the feature sub-set.

$$
\begin{aligned}
& \mathrm{TPR}=\frac{\mathrm{TP}}{\mathrm{TP}+\mathrm{FN}} \\
& \mathrm{FPR}=\frac{\mathrm{FP}}{\mathrm{FP}+\mathrm{TN}}
\end{aligned}
$$

For site-based predictions we sum network scores for residues above a given threshold occurring within a $7 \AA$ region in the protein structure, site-based sensitivity and selectivity was then calculated:

$$
\text { Site selectivity }=\frac{\mathrm{TP}}{\mathrm{TP}+\mathrm{FP}}
$$

where $\mathrm{T}=$ true, $\mathrm{F}=$ false, $\mathrm{P}=$ positive, $\mathrm{N}=$ negative, and $\mathrm{R}=$ rate.

\section{Estimating confidence values}

In order to estimate a confidence value, we analysed the cross-validated network score results of positive and negative sites for each metal ion. Using the $\mathrm{R}$ package, ${ }^{32}$ we calculated the $\log _{10}$ of the ratio of positive cases to negative cases over 20 equal-sized bins along the score range from 0.0 to 1.0. These were plotted, together with their standard errors, and minimum-order polynomial fits were determined. In all cases except copper, fifthorder polynomials gave satisfactory fits to the log ratios when taking their standard errors into account. For copper, a ninth-order polynomial was required to give a satisfactory fit. The resulting equations were used to convert network score outputs into log likelihood ratios for each metal type.

\section{Visualization of metal-site predictions}

The program MetPred was developed to produce a PDB formatted file where the temperature factor column is replaced by the neural network output score. MetPred takes in as inputs the weights obtained from the fully trained classifier and the results can be viewed in any standard molecular graphics viewing program to highlight spatial clusters, indicating likely metal ion interacting residues. All structures in this study were prepared and rendered using the VMD molecular graphics program. ${ }^{33}$

\section{Acknowledgements}

We thank Tim Ebbels, Sundeep Singh Deol and Gurpreet Singh Nagra for helpful discussions. This work was sponsored by the Medical Research Council (to J.S.S. and J.J.W.).

\section{References}

1. Berman, H. M., Westbrook, J., Feng, Z., Gilliland, G., Bhat, T. N., Weissig, H. et al. (2000). The Protein Data Bank. Nucl. Acids Res. 28, 235-242.

2. Orengo, C. A., Michie, A. D., Jones, S., Jones, D. T., Swindells, M. B. \& Thornton, J. M. (1997). CATH: a hierarchic classification of protein domain structure. Structure, 5, 1093-1108.

3. Murzin, A. G., Brenner, S. E., Hubbard, T. \& Chothia,
C. (1995). SCOP: a structural classification of proteins database for the investigation of sequences and structures. J. Mol. Biol. 247, 536-540.

4. Holm, L. \& Sander, C. (1996). Mapping the protein universe. Science, 273, 595-602.

5. Artymiuk, P. J., Poirrette, A., Grindley, H. M., Rice, D. W. \& Willett, P. (1994). A graph-theoretic approach to the identification of three-dimensional pattern of amino acid side-chains in protein structures. J. Mol. Biol. 243, 327-344.

6. Wallace, A. C., Borkakoti, N. \& Thornton, J. M. (1996). TESS: a geometric hashing algorithm for deriving 3D coordinate templates for searching structural databases. Application to enzyme active sites. Protein Sci. 6, 2308-2323.

7. Fetrow, J. S. \& Skolnick, J. (1998). Method for prediction of protein function from sequence using the sequence-to-structure-to-function paradigm with application to glutaredoxins/thioredoxins and $\mathrm{T}_{1}$ ribonucleases. J. Mol. Biol. 281, 949-968.

8. Kleywegt, G. T. (1999). Recognition of spatial motifs in protein structures. J. Mol. Biol. 285, 1887-1897.

9. Bagley, C. B. \& Altman, R. B. (1995). Characterizing the microenviroment surrounding protein site. Protein Sci. 4, 622-935.

10. Wei, L., Huang, E. S. \& Altman, R. B. (1999). Are predicted structures good enough to preserve functional sites? Struct. Fold. Des. 7, 643-650.

11. Altschul, S. F., Madden, T. L., Schäffer, A. A., Zhang, J., Zhang, Z., Miller, W. \& Lipman, D. J. (1997). Gapped BLAST and PSI-BLAST: a new generation of protein database search programs. Nucl. Acids Res. 25, 33893402.

12. Hofmann, K., Bucher, P., Falquet, L. \& Bairoch, A. (1999). The Prosite Database, its status in 1999. Nucl. Acids Res. 27, 215-219.

13. Attwood, T. K., Croning, M. D., Flower, D. R., Lewis, A. P., Mabey, J. E., Scordis, P. et al. (2000). PRINTS-S: the database formerly known as PRINTS. Nucl. Acids Res. 28, 225-227.

14. Bateman, A., Birney, E., Durbin, R., Eddy, S. R., Howe, K. L. \& Sonnhammer, E. (2000). The Pfam protein families database. Nucl. Acids Res. 28, 263-266.

15. Henikoff, J. G., Greene, E. A., Pietrokovoski, S. \& Henikoff, S. (2000). Increased coverage of protein families with the Blocks database servers. Nucl. Acids Res. 28, 228-230.

16. Apweiler, R., Attwood, T., Bairoch, A., Bateman, A., Birney, E., Biswas, M. et al. (2001). The InterPro database, an integrated documentation resource for protein families, domains and functional sites. Nucl. Acids Res. 29, 37-40.

17. Rinaldis, M., Ausiello, G., Cesareni, G. \& HelmerCitterich, M. (1998). Three-dimensional profiles: a new tool of identify protein surface similarities. J. Mol. Biol. 284, 1211-1221.

18. Via, A., Ferre, F., Brannetti, B., Valencia, A. \& HelmerCitterich, M. (2000). Three-dimensional view of the surface motif associated with the P-loop structure: cis and trans cases of convergent evolution. J. Mol. Biol. 303, 1211-1221.

19. Karlin, S., Zhu, Z. Y. \& Karlin, K. D. (1997). The extended environment of mononuclear metal centers in protein structures. Proc. Natl Acad. Sci. USA, 94, 14225-14230.

20. Gregory, D. S., Martin, C. R., Cheetham, J. C. \& Rees, R. A. (1993). The prediction and characterization of metal binding sites in proteins. Protein Eng. 6, 29-35.

21. Rychlewski, L., Fischer, D. \& Elofsson, A. (2003). 
LiveBench-6: large-scale automated evaluation of protein structure prediction servers. Proteins: Struct. Funct. Genet. Supplement 53, 542-547.

22. Thore, S., Mauxion, F., Seraphin, B. \& Suck, D. (2003) $X$-ray structure and activity of the yeast Pop2 protein: a nuclease subunit of the mRNA deadenylase complex. EMBO Rep. 12, 1150-1155.

23. Jones, D. T. (1999). GenTHREADER: an efficient and reliable protein fold recognition method for genomic sequences. J. Mol. Biol. 287, 797-815.

24. McGuffin, L. J. \& Jones, D. T. (2003). Improvement of the GenTHREADER method for genomic fold recognition. Bioinformatics, 19, 874-881.

25. McGuffin, L. J., Street, S., Sorensen, S. \& Jones, D. T. (2004). The genomic threading database. Bioinformatics, 20, 131-132.

26. McGuffin, L. J., Street, S., Bryson, K., Sorensen, S. \& Jones, D. T. (2004). The Genomic Threading Database: a comprehensive resource for structural annotations of the genomes from key organisms. Nucl. Acids Res. 32, D196-D199.

27. Jones, D. T. (1999). Protein secondary structure prediction based on position-specific scoring matrices. J. Mol. Biol. 292, 195-202.
28. Siew, N., Elofsson, A., Rychlewski, L. \& Fischer, D. (2000). MaxSub: an automated measure for the assessment of protein structure prediction quality. Bioinformatics, 16, 776-785.

29. Wang, J., Sykes, B. D. \& Ryan, R. (2002). Structural basis for the conformational adaptability of apolipophorin III, a helix-bundle exchangeable apolipoprotein. Proc. Natl Acad. Sci. USA, 99, 1188-1193.

30. Kabsch, W. \& Sander, C. (1983). Dictionary of protein secondary structure. Pattern recognition and hydrogen-bonded and geometrical features. Biopolymers, 22, 2277-2637.

31. Riedmiller, M. \& Heinrich, B. (1993). A direct adaptive method for faster backpropagation learning: the Rprop algorithm. Proceedings of the ICNN.

32. Ihaka, R. \& Gentleman, R. (1996). R: a language for data analysis and graphics. J. Comput. Graph. Stat. 5, 299-314.

33. Humphrey, W., Dalke, A. \& Schulten, K. (1996). VMD—visual molecular dynamic. J. Mol. Graph. 14, 33-38.

Edited by J. Thornton

(Received 2 April 2004; received in revised form 6 July 2004; accepted 8 July 2004) 